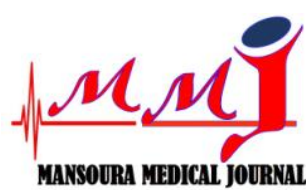

Since 1987 ISSN: 1110-211X
Mansoura IMedical Journal

(Official Journal of Mansoura Faculty of Medicine)

pISSN: 1110-211X; eISSN: 2735-3990

\title{
Use of forearm basilic vein as an option of upper limb vascular access for haemodialysis
}

\author{
By
}

\section{Mohamed Ashraf Elsayed Elshazli, Samer Abdelhamid Regal MD , Hossam Mohamed Mustafa MD, Amr M. Elshafei.}

\section{Vascular Surgery department Mansoura University, Egypt.}

DOI : $10.21608 / \mathrm{mimu} .2021 .81651 .1019$

Submit Date: June 1, 2021

Accept Date : June 30, 2021

Available online: July 30, 2021

\section{Keywords}

- $\quad$ Ckd

- Dialysis

- access.

\begin{abstract}
in our study, we aimed to evaluate the outcome of forearm basilc vein transposition and anastomosis to either radial or brachial areteries to create arteriovenous fistula for haemodialysis. Patient and Methods : This is an observational prospective study conducted from august 2019 to December 2020 at the Department of Vascular Surgery, Mansoura University hospital . 25 patients were selected for forearm basilic vein transposition (FBVT ) . 18 cases underwemt BB loop AVF and 7 cases underwent radiobasilic AVF . procedure was done under combined supraclavicular and local xylocaine $1 \%$ anaethesia. Basilic vein was harvested using multiple incisions then elevated and anastmosed to either radial artery in 7 cases or tunneled as aloop and anastomsed to brachial artery in 18 cases . Results: The mean age of patients who received an AVF was 56.96 \pm 9.61 years, with a range of 35 to 70 years, and included 19 men (76\%) and 6 women (24\%).18 cases underwemt BB looped AVF and 7 cases underwent radiobasilic AVF . patients were followed up for 1 year. 1ry patency rates at 3,6,12 months were $84 \%, 83.3 \%, 82.6 \%$. Conclusion: Radiobasilic or forearm brachiobasilic looped AVF can be used as an alternative option in case of cephalic vein inadequancy or if it was previously used for haemdialysis.
\end{abstract}




\section{INTRODUCTION}

It is essential to secure and maintain adurable vascular access with minimal risk of complications for proper dialysis in patients on chronic regular hemodialysis. ${ }^{1}$

Autogenous arteriovenous fistulas (AVF) are the preferred mode of vascular access for maintenance hemodialysis (HD) followed by prosthetic grafts and finally central permenant catheters ${ }^{1,2,6}$.

The Kidney Disease Outcome Quality Initiative (KDOQI) recommends autologous radiocephalic or brachiocephalic AVF as a primary method of choice in HD patients. the basilic vein transposition (BVT) comes as a secondary option in patients with failed radio-cephalic fistula (RCF) or brachiocephalic fistula $(\mathrm{BCF}) .^{3}$

Arm brachiobasilic AVF was widely discussed by many groups either by one stage transposition or by two stages . but fewer studies have been discussing FBVT, although its valuable role and relatively old route. $^{4}$

Forearm basilic vein could be used before arm basilic vein transposition, providing another option for autogenous hemodialysis access. ${ }^{5}$

In patients with unfavourable anatomy, a looped transposition of forearm basilic vein (BV) can be created to facilitate more proximal anastomosis with brachial, radial or ulnar artery in the antecubital region. ${ }^{7}$

\section{Patient and methods}

This is an observational prospective study conducted from august.2019 to December 2020 at the vascular surgery department, Mansoura University hospitals .

ERDS patients with Cephalic vein inadequacy (upper arm and forearm) which my be Previously used for access or Destroyed by venipuncture or Unsuitable anatomy are considered in our study . Duplex us of venous system of both upper limbs was examined starting with cephalic vein. In case of inadequate cephalic vein, basilic vein was examined. Forearm basilic vein Diameter which should be $>2.5 \mathrm{~mm}$ in dimater and adequetly distensible with patency throughout its course . Arm basilic vein should also be examined which should be patent with $3 \mathrm{~mm}$ or more in diameter with adequate distensibility up to axilla .

Color DUS was conducted on Arterial vessels of the arm from axillary to radial and ulnar artery. Arterial vessels examinations consists of evaluating diameter, wall morphology and PSV.

The radial artery in the forearm should be of 2 $\mathrm{mm}$ or more in diameter and brachial artery should be of $4 \mathrm{~mm}$ or more with no signs of vasculopathy as arterial calcifications and/or stenosis.

When radial artery is suitable to use, basilic vein was transposed and anastmosed to radial artery . in case of unsuitability of radial artery, forearm basilic vein was transposed and tunneled as aloop and anastmosed to brachial artery .

25 patients were selected for FBVT . The mean age of patients who received an AVF was $56.96 \pm 9.61$ years, with a range of 35 to 70 years, and included 19 men (76\%) and 6 women (24\%). 3 cases had previous radiocephalic AVF and 2 cases had previous brachiocephalic AVF which were thrombosed . other 20 cases had inadequate cephalic vein .18 cases underwent BB looped AVF .7 cases underwent radiobasilc AVF . basilic vein diameter in studied cases was $3.06 \pm 0.29 \mathrm{~mm}$. 
Patients were examined clinically by Allen's test to assess palmar arch patency and arterial pulsations were detected and skin marked . forearm baslic vein was examined clinicaly under tourniquet to assess patency and its continuity throughout forearm to elbow. Duplex us was conducted on areterial and venous system of both upper limbs with mapping of FBV to confirm patency and size of forearm basilic vein which decreased surgical exposure and dissection times.

\section{Surgical technique :}

Preoperative mapping of forearm Basilic vein through physical examination and Doppler ultrasound was mandatory in all cases. All Patients were operated using supraclavicular anaesthesia while 10 patients required combined supraclavicular and local xylocaine $1 \%$ anaethesia. We used to perform three or four separate longitudinal skin incisions to dissect FBV from the elbow to the wrist region . Tributaries were ligated using absorbalble vicryl 3/0 sutures . Then basilic vein was ligated and divided distally for its elevation. Then baslic vein was inflated with heparinized saline and thrill was palpated through the vein to ensure its patency .

in 7 patients, radial artery ( had adiameter ranging from $2 \mathrm{~mm}$ to $3.2 \mathrm{~mm}$ with no calcifications or stenosis with PSV ranged from 40 to $80 \mathrm{~cm} / \mathrm{sec}$ ) was dissected and exposed above wrist and prepared for anastomosis. A subcutaneous tunnel on the ventral aspect of the forearm was created . then $\mathrm{BV}$ was anastomsed end to side to radial artery with polyprolene $6 / 0$ sutures . While in 18 patients, radial artery was unsuitable, brachial artery was examined which had adiameter ranged from $3.5 \mathrm{~mm}$ to $5 \mathrm{~mm}$ with PSV ranged from 45 to $90 \mathrm{~cm} / \mathrm{sec}$. It was exposed at cubital fossa then
BV was tunneled subcutaneously as aloop and anastmosed end to side to BA . care was taken to avoid twisting of vein .Vascular bulldog clamp was applied proximally at basilic vein . separate incisions were made to allow transposition of vein while it is inflated with heparinized saline. operative time was approximately $100 \mathrm{~min}$.

\section{Results:}

The mean age of patients who received an AVF was $56.96 \pm 9.61$ years, with a range of 35 to 70 years, and included 19 men (76\%) and 6 women (24\%).13 DM, 18HTN,4cardiac,4smokers ,9 with hyperlipedemia , 2hepatic, 17 patients were on haemodialysis , 5 patients had previous AVF (3 RCAVF, 2BC AVF ).18 cases underwemt BB loop $\mathrm{AVF}$ and 7 cases underwent radiobasilic AVF . table 1,2 .

Successful maturation occurs in 22 patients. Maturation timing was approximately 44.96 days . 7 cases had radiobasilic AVF . among them one case failed to mature and underwent successful venoplasty . 18 cases had BB loope AVF . among them 2 cases failed to mature . one of them underwent another access ( arm BB AVF ) the other one underwent superfiscialization of arm basilic vein. Basilic vein thrombosis was reported after 3months in 1 patient with BB loop and underwent successful thrombectomy.

Death was reported in 2 cases after 4, 6 months of creation of AVF. 4 cases developed sc edema they were managed conservatively and edema resolved completely. 3 cases developed seroma at harvesting wound which resolved spontaneously 1 wk later. 2 cases had small hematomas at harvesting wounds . they were managed by hot fomentation and daily dressing. There were no 
reported cases of venous hypertension, steel syndrome or infections . table 3,4.

\section{Patency rates:}

patients were followed up for 1 year .Regular examination was done at 1,3,6,12 months post operative. 1ry patency rates at 3,6,12 months were $84 \%, 83.3 \%, 82.6 \%$.

Table 5 , figure 8,9 .

1ry patency rate at 12 months was $85.7 \%$ for $\mathrm{RB}$ AVF and $81.25 \%$ for BB looped AVF .table 10 .

Table (1):demographic characteristics of the studied cases.

\begin{tabular}{|l|l|l|}
\hline \multicolumn{1}{|c|}{$\mathbf{N}=25$} & $\%$ \\
\hline $\begin{array}{l}\text { Age/years } \\
\text { mean+SD (min-max) }\end{array}$ & $56.96 \pm 9.61(35-70)$ & \\
\hline $\begin{array}{l}\text { Sex } \\
\text { Male }\end{array}$ & 19 & 76.0 \\
Female & 6 & 24.0 \\
\hline $\begin{array}{l}\text { Occupation } \\
\text { Not working } \\
\text { Working }\end{array}$ & 10 & 40.0 \\
\hline
\end{tabular}

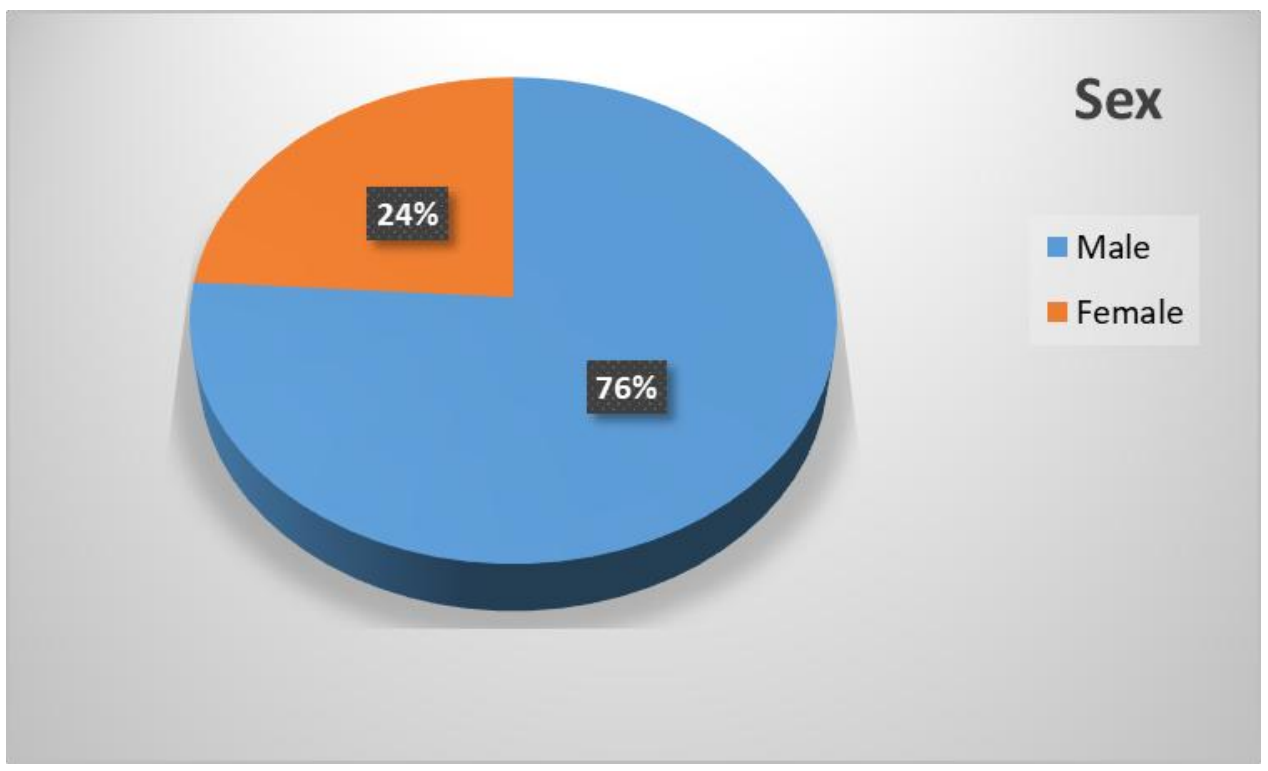

figure (1): sex of the studied cases.

Table (2): medical history of the studied cases.

\begin{tabular}{|l|l|l|}
\hline DM & $\mathbf{N}=\mathbf{2 5}$ & \% \\
\hline Hypertension & 13 & 52.0 \\
\hline Cardiac & 18 & 72.0 \\
\hline Smoking & 4 & 16.0 \\
\hline Hyperlipidemia & 4 & 16.0 \\
\hline Hepatic & 9 & 36.0 \\
\hline Dialysis & 2 & 8.0 \\
\hline Previous Fistula & 17 & 68.0 \\
\hline
\end{tabular}




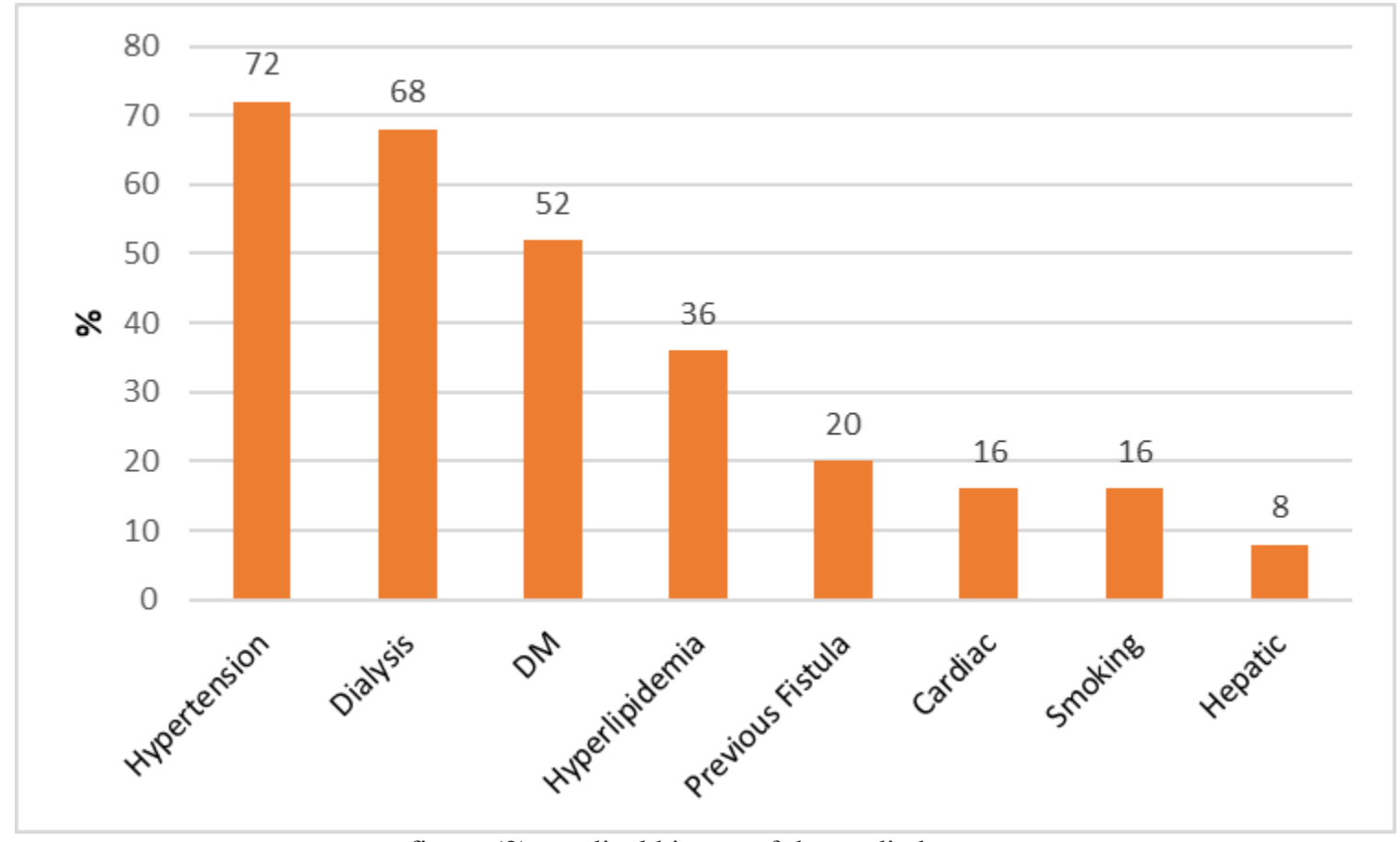

figure (2): medical history of the studied cases

Table (3): Operation characteristics distribution among studied cases

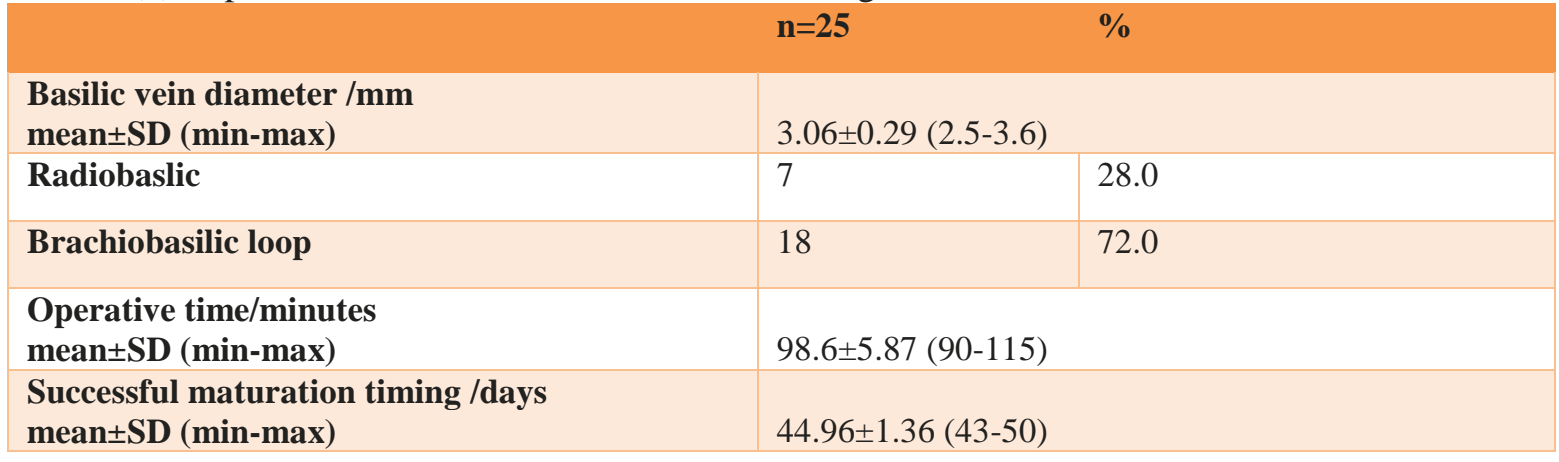

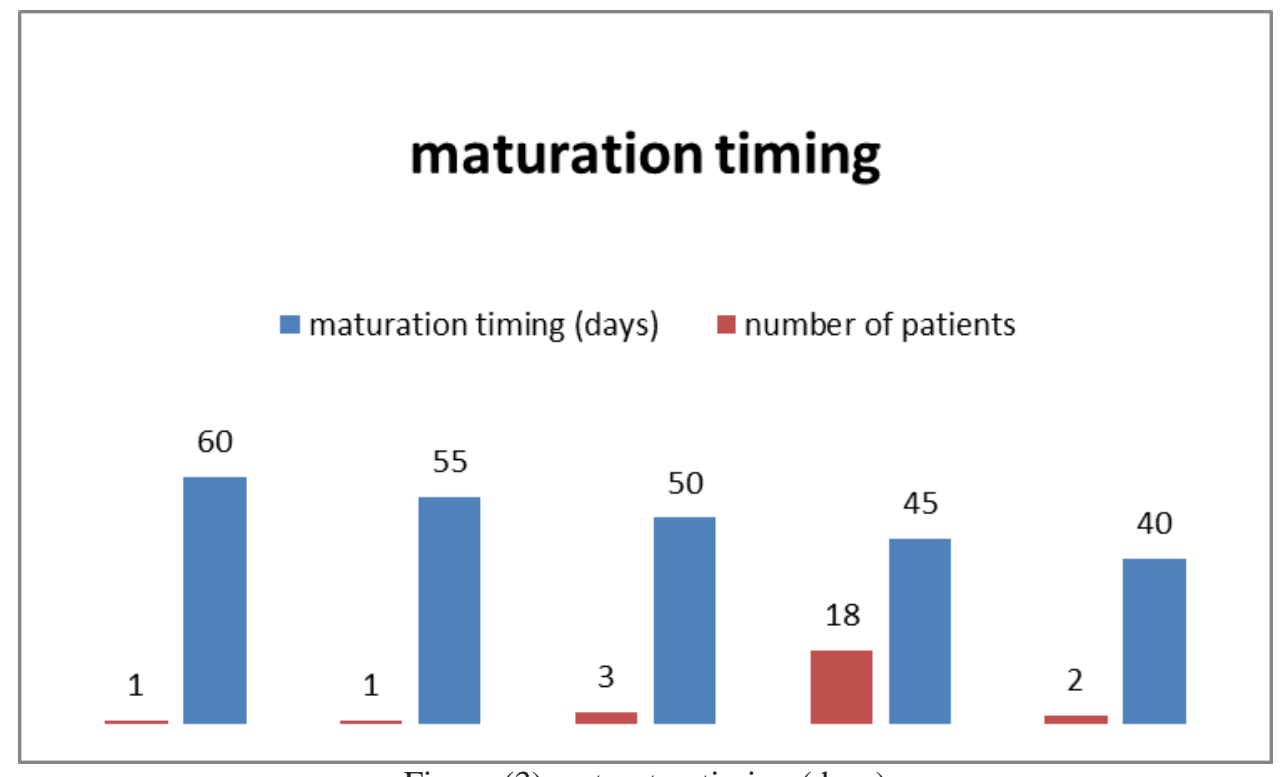

Figure (3) maturaton timing (days) 


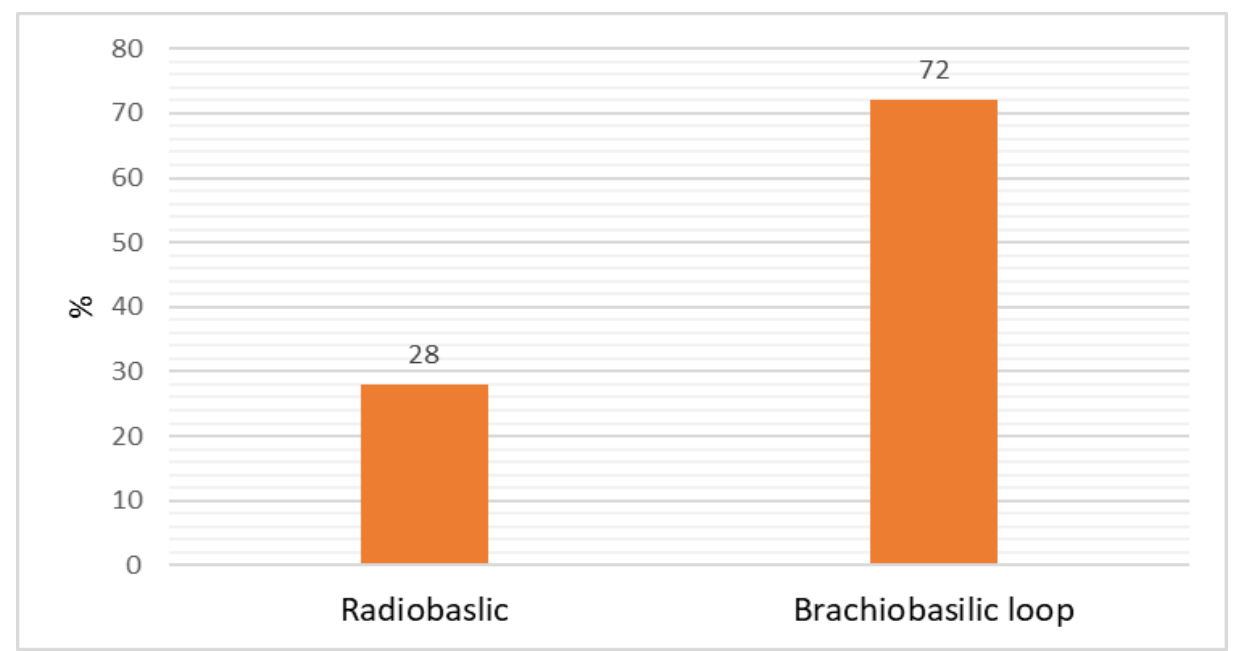

Figure (4) \% of RB vs BB loop

\section{successful maturation}

- successful maturation

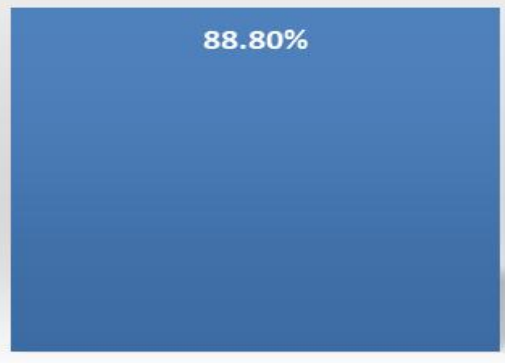

BRACHIOBASILIC LOOP(18 cases )

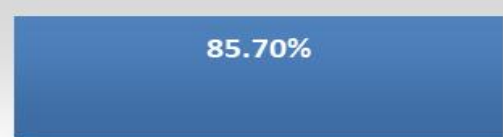

RADIOBASILIC(7 cases)

Figure (5) maturation success

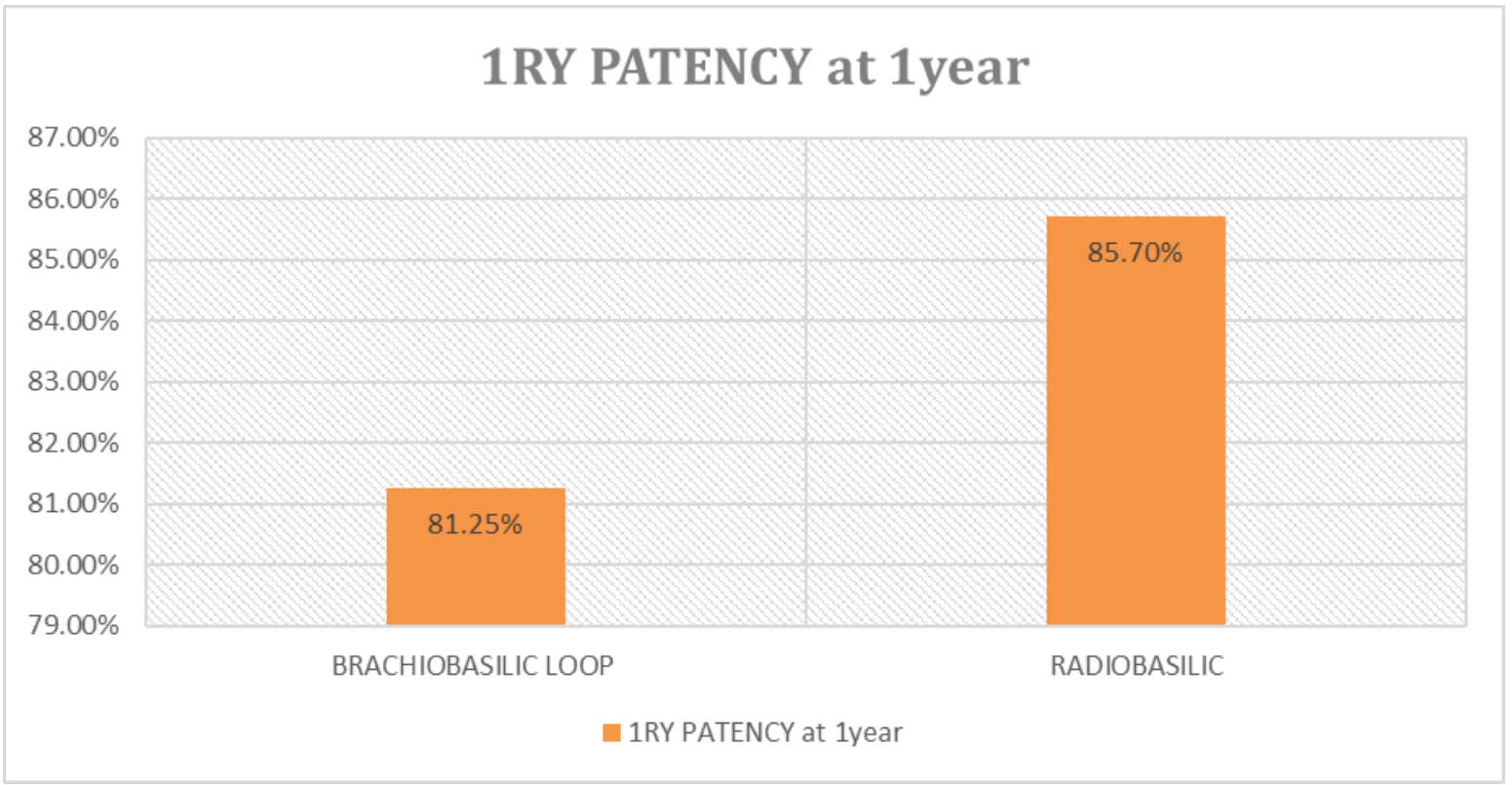

Figure (6) Iry patency rate at 1 year 
Table (4): complications distribution among studied cases.

\begin{tabular}{|l|c|c|}
\hline & $\mathbf{n = 2 5}$ & $\mathbf{\%}$ \\
\hline Infection & 0 & 0.0 \\
\hline Hematoma & 2 & 8.0 \\
\hline Seroma & 3 & 12.0 \\
\hline Thrombosis & 1 & 4.0 \\
\hline SC oedema & 4 & 16.0 \\
\hline Venous HTN & 0 & 0.0 \\
\hline Steal syndrome & 0 & 0.0 \\
\hline stenosis(N=23) & 1 & 4.0 \\
\hline Maturation failure & 3 & 12.0 \\
\hline
\end{tabular}

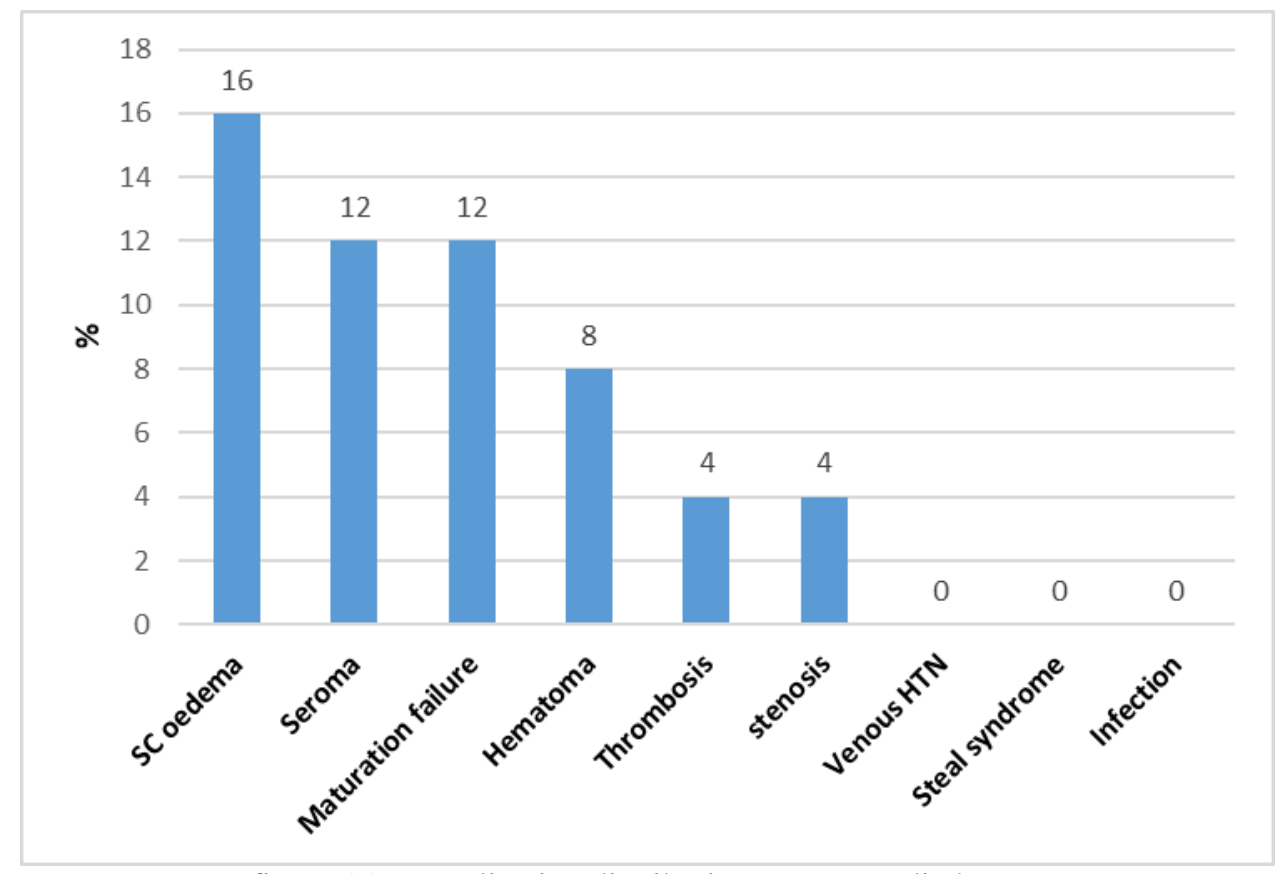

figure (7): complication distribution among studied cases

Table (5): outcome distribution among studied cases.

\begin{tabular}{|c|c|c|}
\hline & $\mathrm{n}=\mathbf{2 5}$ & $\%$ \\
\hline Death & 2 & 8.0 \\
\hline Patency at 1m & 22 & 88.0 \\
\hline Patency at 3m & 21 & 84.0 \\
\hline Patency at $6 \mathrm{~m}(\mathrm{~N}=24)$ & 20 & 83.3 \\
\hline Patency at $12 \mathrm{~m}(\mathrm{~N}=\mathbf{2 3})$ & 19 & 82.6 \\
\hline Intervention venoplasty $(\mathrm{N}=\mathbf{2 3})$ & 1 & 4.3 \\
\hline Superficialization of arm BB $(\mathrm{N}=23)$ & 1 & 4.3 \\
\hline Thrombectomy $(\mathrm{N}=23)$ & 1 & 4.3 \\
\hline Another access & $\mathrm{N}=23$ & \\
\hline$-\mathrm{VE}$ & 21 & 91.3 \\
\hline Superficiliazation & 1 & 4.3 \\
\hline $\operatorname{arm} B B \mathrm{AVF}$ & 1 & 4.3 \\
\hline Secondary patency & $\mathrm{N}=4 *$ & \\
\hline lost access (arm BB AVF) & 1 & 25.0 \\
\hline Patent & 3 & 75.0 \\
\hline
\end{tabular}




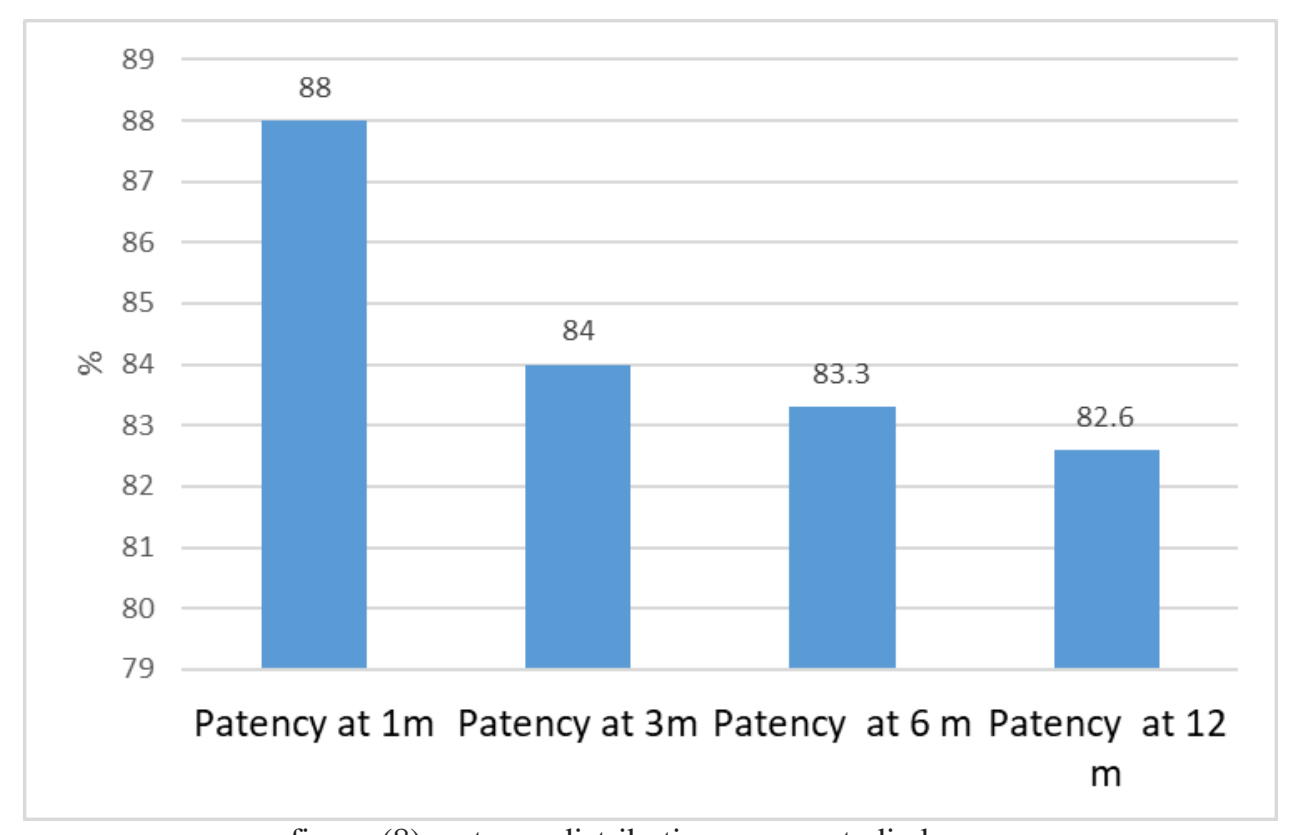

figure (8): patency distribution among studied cases

Table (6):Association between incidence of patency at one month of follow up and studied risk factors among studied cases.

\begin{tabular}{|c|c|c|c|c|}
\hline & \multirow{2}{*}{$\begin{array}{c}\text { Total } \\
\text { number } \\
=25\end{array}$} & \multicolumn{2}{|c|}{ Patency at $1 \mathrm{~m}$} & \multirow{2}{*}{ Test of significance } \\
\hline & & $\begin{array}{l}-V E \\
n=3\end{array}$ & $\begin{array}{l}+V E \\
n=22\end{array}$ & \\
\hline Age/years & 25 & $64.0 \pm 5.65$ & $56.35 \pm 9.71$ & $\mathrm{t}=1.08, \mathrm{p}=0.289$ \\
\hline $\begin{array}{l}\text { Sex } \\
\text { Male } \\
\text { Female }\end{array}$ & $\begin{array}{c}19 \\
6\end{array}$ & $\begin{array}{c}3(15.8) \\
0\end{array}$ & $\begin{array}{c}16(84.2) \\
6(100)\end{array}$ & FET, $\mathrm{P}=0.554$ \\
\hline $\begin{array}{l}\text { Occupation } \\
\text { Not working } \\
\text { Working }\end{array}$ & $\begin{array}{l}10 \\
15\end{array}$ & $\begin{array}{l}2(20) \\
1(6.7)\end{array}$ & $\begin{array}{c}8(80) \\
14(93.3)\end{array}$ & FET, $\mathrm{P}=0.543$ \\
\hline DM & 13 & $3(23.1)$ & $10(76.9)$ & FET, $\mathrm{P}=0.22$ \\
\hline Hypertension & 18 & $2(11.1)$ & $16(88.9)$ & FET, $\mathrm{P}=1.0$ \\
\hline Cardiac & 4 & $1(25)$ & $3(75)$ & FET, $\mathrm{P}=0.422$ \\
\hline Smoking & 4 & 0 & $4(100)$ & FET,$P=1.0$ \\
\hline Hyperlipidemia & 9 & $2(22.2)$ & $7(77.8)$ & $\begin{array}{c}\text { FET } \\
\mathrm{P}=0.53\end{array}$ \\
\hline Hepatic & 2 & 0 & $2(100)$ & FET, $\mathrm{P}=1.0$ \\
\hline Dialysis & 17 & $3(17.6)$ & $14(82.4)$ & FET, $P=0527$ \\
\hline Previous Fistula & 5 & 0 & $5(100)$ & FET, $\mathrm{P}=1.0$ \\
\hline Basilic vein diameter /mm & 25 & $2.55 \pm 0.07$ & $3.10 \pm 0.267$ & $\begin{array}{c}\mathrm{t}=2.87 \\
\mathrm{p}=0.009^{*}\end{array}$ \\
\hline Radiobaslic & 7 & $1(14.3)$ & $6(85.7)$ & FET, $\mathrm{P}=1.0$ \\
\hline Brachiobasilic loop & 18 & $2(11.1)$ & $16(88.9)$ & FET,$P=1.0$ \\
\hline Operative time/minutes & 25 & $100 \pm 0$ & $98.48 \pm 6.11$ & $\mathrm{t}=0.345, \mathrm{p}=0.733$ \\
\hline Successful maturation timing /days & 25 & & $44.96 \pm 1.36$ & \\
\hline Hematoma & 2 & 0 & $2(100)$ & FET, $\mathrm{P}=1.0$ \\
\hline Seroma & 3 & 0 & $3(100)$ & FET, $\mathrm{P}=1.0$ \\
\hline Thrombosis & 1 & $1(100)$ & 0 & FET , $\mathrm{P}=0.12$ \\
\hline SC oedema & 4 & 0 & $4(100)$ & FET, $\mathrm{P}=1.0$ \\
\hline Maturation failure & 3 & $3(100)$ & $0(0)$ & FET, $\mathrm{P}=0.01 *$ \\
\hline
\end{tabular}

FET :Fischer exact test , $\mathrm{t}:$ Student $\mathrm{t}$ test , *statistically significant if $\mathrm{p}<0.05$ 
Table (7):Association between incidence of patency at 3 months of follow up and studied risk factors among studied cases.

\begin{tabular}{|c|c|c|c|c|}
\hline & \multirow{2}{*}{$\begin{array}{c}\begin{array}{c}\text { Total } \\
\text { number } \\
=25\end{array} \\
\text { = }\end{array}$} & \multicolumn{2}{|c|}{ Patency at 3 m } & \multirow{2}{*}{ Test of significance } \\
\hline & & $\begin{array}{c}-\mathrm{VE} \\
\mathrm{n}=4(16.0 \%)\end{array}$ & $\begin{array}{c}+\mathrm{VE} \\
\mathrm{n}=21(84.0 \%)\end{array}$ & \\
\hline Age/years & 25 & $65.50 \pm 4.04$ & $55.33 \pm 9.54$ & $\mathrm{t}=2.08, \mathrm{p}=0.05$ \\
\hline $\begin{array}{l}\text { Sex } \\
\text { male } \\
\text { female }\end{array}$ & $\begin{array}{c}19 \\
6\end{array}$ & $\begin{array}{l}3(15.8) \\
1(16.7)\end{array}$ & $\begin{array}{c}16(84.2) \\
5(83.3)\end{array}$ & FET,$P=1.0$ \\
\hline $\begin{array}{l}\text { Occupation } \\
\text { Not working } \\
\text { Working }\end{array}$ & $\begin{array}{l}10 \\
15\end{array}$ & $\begin{array}{c}3(30.0) \\
1(6.7)\end{array}$ & $\begin{array}{c}7(70.0) \\
14(93.3)\end{array}$ & FET, $P=0.267$ \\
\hline DM & 13 & $3(23.1)$ & $10(76.9)$ & FET, $\mathrm{P}=0.593$ \\
\hline Hypertension & 18 & $3(16.7)$ & $15(83.3)$ & FET, $\mathrm{P}=1.0$ \\
\hline Cardiac & 4 & $2(50.0)$ & $2(50.0)$ & FET, $\mathrm{P}=0.106$ \\
\hline Smoking & 4 & 0 & $4(100)$ & FET, $P=1.0$ \\
\hline Hyperlipidemia & 9 & $3(33.3)$ & $6(66.7)$ & FET, $P=0.116$ \\
\hline Hepatic & 2 & $0(0.0)$ & $2(100)$ & FET, $\mathrm{P}=1.0$ \\
\hline Dialysis & 17 & $4(23.5)$ & $13(76.5)$ & FET, $P=0.269$ \\
\hline Previous Fistula & 5 & $1(20.0)$ & $4(80.0)$ & FET, $\mathrm{P}=1.0$ \\
\hline Basilic vein diameter /mm & 25 & $2.63 \pm 0.125$ & $3.14 \pm 0.24$ & $\mathrm{t}=4.09, \mathrm{p}<0.001 *$ \\
\hline Radiobaslic & 7 & $1(14.3)$ & $6(85.7)$ & $\mathrm{FET}, \mathrm{P}=1.0$ \\
\hline Brachiobasilic loop & 18 & $3(16.7)$ & $15(83.3)$ & FET, $\mathrm{P}=1.0$ \\
\hline Operative time/minutes & 25 & $100 \pm 0.0$ & $98.33 \pm 6.39$ & $\mathrm{t}=0.513, \mathrm{p}=0.613$ \\
\hline Successful maturation timing /days & 25 & $47.50 \pm 3.54$ & $44.71 \pm 0.845$ & $\mathrm{t}=3.33, \mathrm{p}=0.003^{*}$ \\
\hline Hematoma & 2 & $1(50)$ & $1(50)$ & FET, $\mathrm{P}=0.30$ \\
\hline Seroma & 3 & $1(33.3)$ & $2(66.7)$ & FET, $\mathrm{P}=0.422$ \\
\hline Thrombosis & 1 & $1(100)$ & $0(0.0)$ & FET, $P=0.160$ \\
\hline SC oedema & 4 & 0 & $4(100)$ & FET, $\mathrm{P}=1.0$ \\
\hline Maturation failure & 3 & $3(100)$ & 0 & FET, $\mathrm{P}=0.002 *$ \\
\hline
\end{tabular}

FET :Fischer exact test $, \mathrm{t}:$ Student $\mathrm{t}$ test,${ }^{*}$ statistically significant if $\mathrm{p}<0.05$

Table (8):Association between incidence of patency at 6 months of follow up and studied risk factors among studied cases.

\begin{tabular}{|c|c|c|c|c|}
\hline & \multirow{2}{*}{$\begin{array}{l}\text { Total } \\
\text { number } \\
=24\end{array}$} & \multicolumn{2}{|c|}{ Patency at 6 months } & \multirow[t]{2}{*}{ Test of significance } \\
\hline & & $\begin{array}{c}-\mathrm{VE} \\
\mathrm{N}=4(16.7 \%)\end{array}$ & $\begin{array}{c}+\mathrm{VE} \\
\mathrm{N}=20(83.3 \%)\end{array}$ & \\
\hline Age/years & 24 & $64.0 \pm 5.66$ & $55.73 \pm 9.46$ & $\mathrm{t}=1.20, \mathrm{p}=0.242$ \\
\hline $\begin{array}{l}\text { Sex } \\
\text { Male } \\
\text { Female }\end{array}$ & $\begin{array}{c}19 \\
5\end{array}$ & $\begin{array}{c}3(15.8) \\
1(20)\end{array}$ & $\begin{array}{c}16(84.2) \\
4(80)\end{array}$ & FET, $\mathrm{P}=1.0$ \\
\hline $\begin{array}{l}\text { Occupation } \\
\text { Not working } \\
\text { Working }\end{array}$ & $\begin{array}{l}10 \\
14\end{array}$ & $\begin{array}{l}3(30) \\
1(7.1)\end{array}$ & $\begin{array}{c}7(70.0) \\
13(92.9)\end{array}$ & FET, $P=0.272$ \\
\hline DM & 12 & $3(25)$ & $9(75)$ & FET, $\mathrm{P}=0.590$ \\
\hline Hypertension & 17 & $3(17.6)$ & $14(82.4)$ & FET, $P=1.0$ \\
\hline Cardiac & 4 & $2(50)$ & $2(50)$ & FET, $\mathrm{P}=1.0$ \\
\hline Smoking & 4 & 0 & $4(100)$ & $\mathrm{FET}, \mathrm{P}=1.0$ \\
\hline Hyperlipidemia & 8 & $3(37.5)$ & $5(62.5)$ & FET, $\mathrm{P}=0.09$ \\
\hline Hepatic & 2 & $0(0.0)$ & $2(100)$ & $\mathrm{FET}, \mathrm{P}=1.0$ \\
\hline Dialysis & 16 & $4(25.0)$ & $12(75.0)$ & FET, $P=0.262$ \\
\hline Previous Fistula & 5 & $1(20)$ & $4(80)$ & FET, $\mathrm{P}=1.0$ \\
\hline Basilic vein diameter /mm & 24 & $2.55 \pm 0.07$ & $3.10 \pm 0.27$ & $\mathrm{t}=2.79, \mathrm{p}=0.01 *$ \\
\hline Radiobaslic & 7 & $1(14.3)$ & $6(85.7)$ & FET, $\mathrm{P}=1.0$ \\
\hline Brachiobasilic loop & 17 & $3(17.6)$ & $14(82.4)$ & FET, $\mathrm{P}=1.0$ \\
\hline Operative time/minutes & 24 & $100 \pm 0.0$ & $97.73 \pm 5.05$ & $\mathrm{t}=0.623, \mathrm{p}=0.540$ \\
\hline Successful maturation timing /days & 24 & & $45.05 \pm 1.33$ & \\
\hline Hematoma & 2 & $1(50)$ & $1(50)$ & $\mathrm{FET}, \mathrm{P}=1.0$ \\
\hline Seroma & 3 & $1(25)$ & $2(75)$ & FET, $\mathrm{P}=0.437$ \\
\hline Thrombosis & 1 & $1(100)$ & $0(0.0)$ & FET, $\mathrm{P}=0.167$ \\
\hline SC oedema & 3 & 0 & $3(100)$ & FET, $\mathrm{P}=1.0$ \\
\hline Maturation failure & 3 & $3(100)$ & $0(0.0)$ & FET, $\mathrm{P}=0.002 *$ \\
\hline
\end{tabular}

FET :Fischer exact test, $\mathrm{t}$ :Student $\mathrm{t}$ test, *statistically significant if $\mathrm{p}<0.05$ 
Table (9):Association between incidence of patency at 12 months and studied risk factors among studied cases.

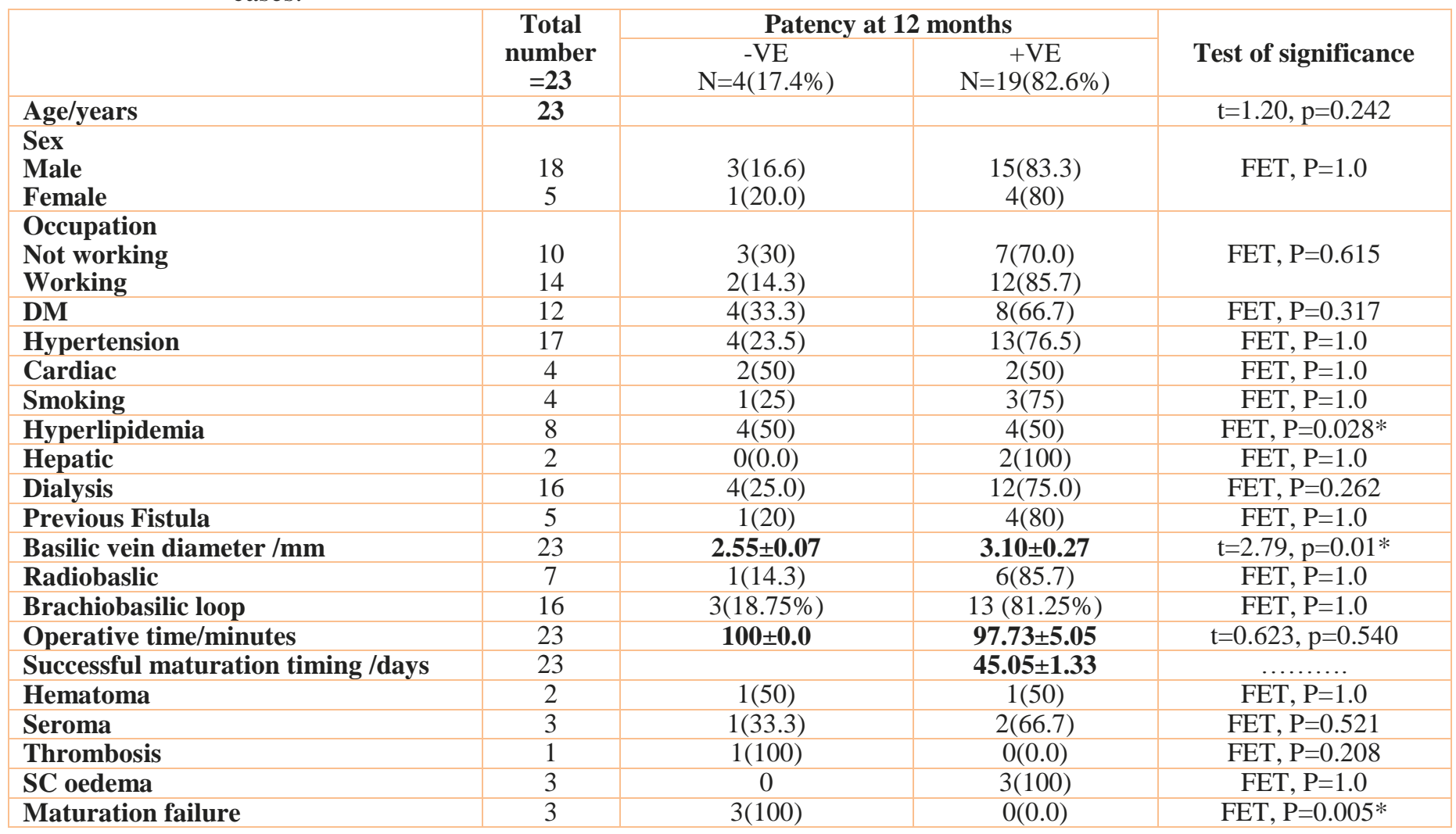

FET :Fischer exact test , $\mathrm{t}:$ Student $\mathrm{t}$ test, *statistically significant if $\mathrm{p}<0.05$

Table(10): Association between incidence of patency at 12 months and studied risk factors among RB and BB looped AVF

\begin{tabular}{|c|c|c|c|c|}
\hline & $\begin{array}{c}\text { Total number } \\
=25\end{array}$ & & & of significance \\
\hline Maturation failure & 3 & $2(11.1)$ & $1(14.3)$ & $\begin{array}{c}\text { FET } \\
\mathrm{P}=1.0\end{array}$ \\
\hline basilic.vein.diameter.MM & & $3.10 \pm 0.30$ & $2.95 \pm 0.28$ & $\begin{array}{c}\mathrm{t}=1.08 \\
\mathrm{p}=0.292\end{array}$ \\
\hline operative.time.min & & $100.83 \pm 4.62$ & $92.85 \pm 4.87$ & $\begin{array}{c}\mathrm{t}=3.82 \\
\mathrm{p}=0.001 *\end{array}$ \\
\hline successful_maturation_timing & & $44.56 \pm 0.89$ & $45.86 \pm 1.86$ & $\begin{array}{c}\mathrm{t}=2.28 \\
\mathrm{p}=0.03^{*}\end{array}$ \\
\hline SC oedema & 4 & $2(11.1)$ & $2(28.6)$ & $\begin{array}{c}\text { FET } \\
\mathrm{P}=0.548\end{array}$ \\
\hline Hematoma & 2 & $2(11.1)$ & 0 & $\begin{array}{l}\text { FET } \\
\mathrm{P}=1.0\end{array}$ \\
\hline Seroma & 3 & $2(11.1)$ & $1(14.3)$ & $\begin{array}{c}\text { FET } \\
\mathrm{P}=1.0\end{array}$ \\
\hline Thrombosis & 1 & $1(5.6)$ & 0 & $\begin{array}{c}\text { FET } \\
\mathrm{P}=1.0\end{array}$ \\
\hline Primary patency at 12 months & 19 & $13(81.25)$ & $6(85.7)$ & $\begin{array}{l}\text { FET } \\
\mathrm{P}=1.0\end{array}$ \\
\hline Intervention venoplasty & 1 & 0 & $1(14.3)$ & $\begin{array}{c}\text { FET } \\
\mathrm{P}=1.0\end{array}$ \\
\hline Superficializing of arm BB & 1 & $1(5.6)$ & 0 & $\begin{array}{c}\text { FET } \\
\mathrm{P}=1.0\end{array}$ \\
\hline Thrombectomy & 1 & $1(5.6)$ & 0 & $\begin{array}{c}\text { FET } \\
\mathrm{P}=1.0\end{array}$ \\
\hline $\begin{array}{l}\text { Another access } \\
\text { 0 } \\
\text { superficialization } \\
\text { arm BB AVF }\end{array}$ & $\begin{array}{c}23 \\
1 \\
1\end{array}$ & $\begin{array}{c}16(88.9) \\
1(5.6) \\
1(5.6)\end{array}$ & $\begin{array}{c}7(100) \\
0 \\
0\end{array}$ & $\begin{array}{c}\mathrm{MC} \\
\mathrm{P}=0.655\end{array}$ \\
\hline
\end{tabular}




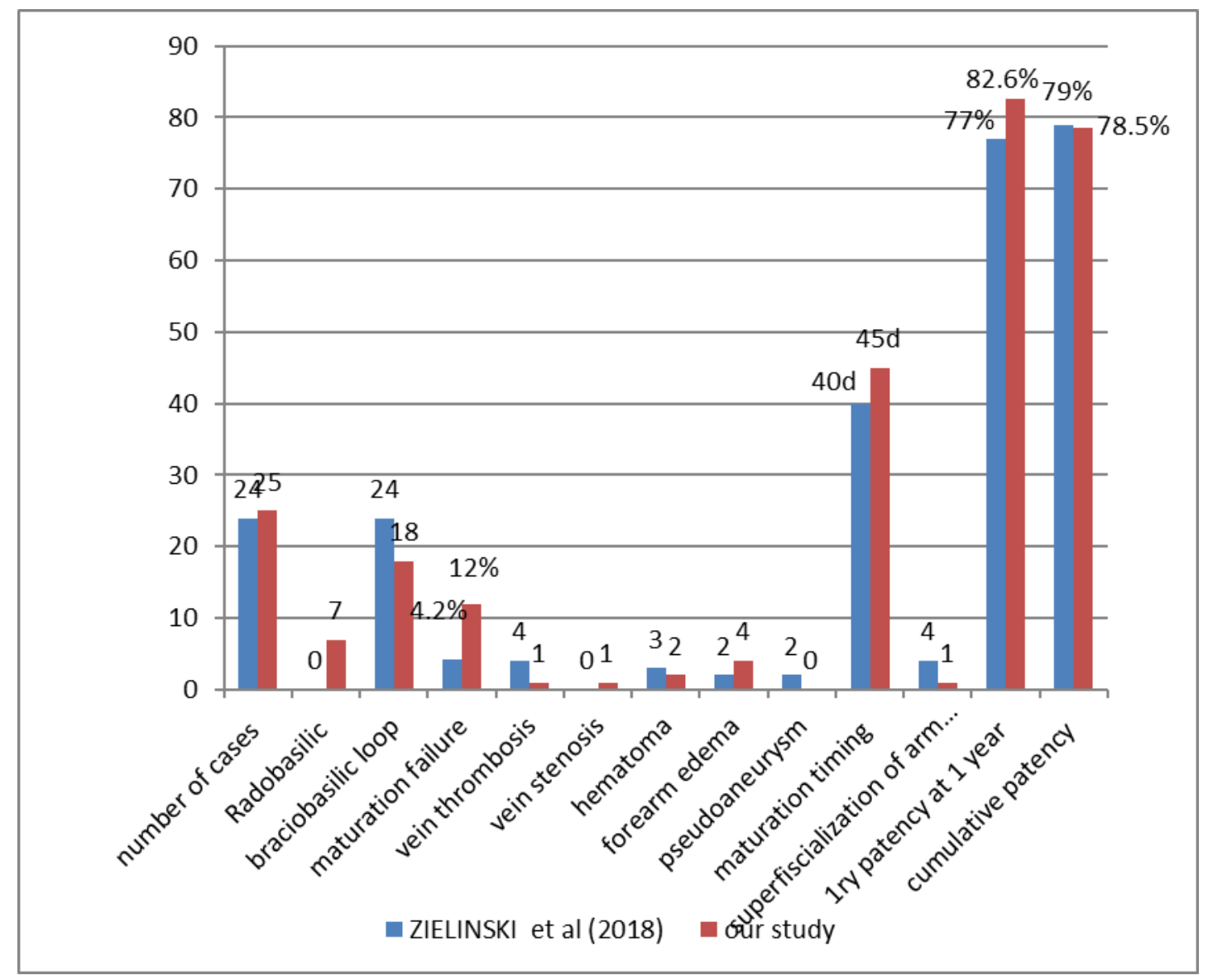

Figure (10) : comparison between Zeilinski et al(BB loop ) and our study .
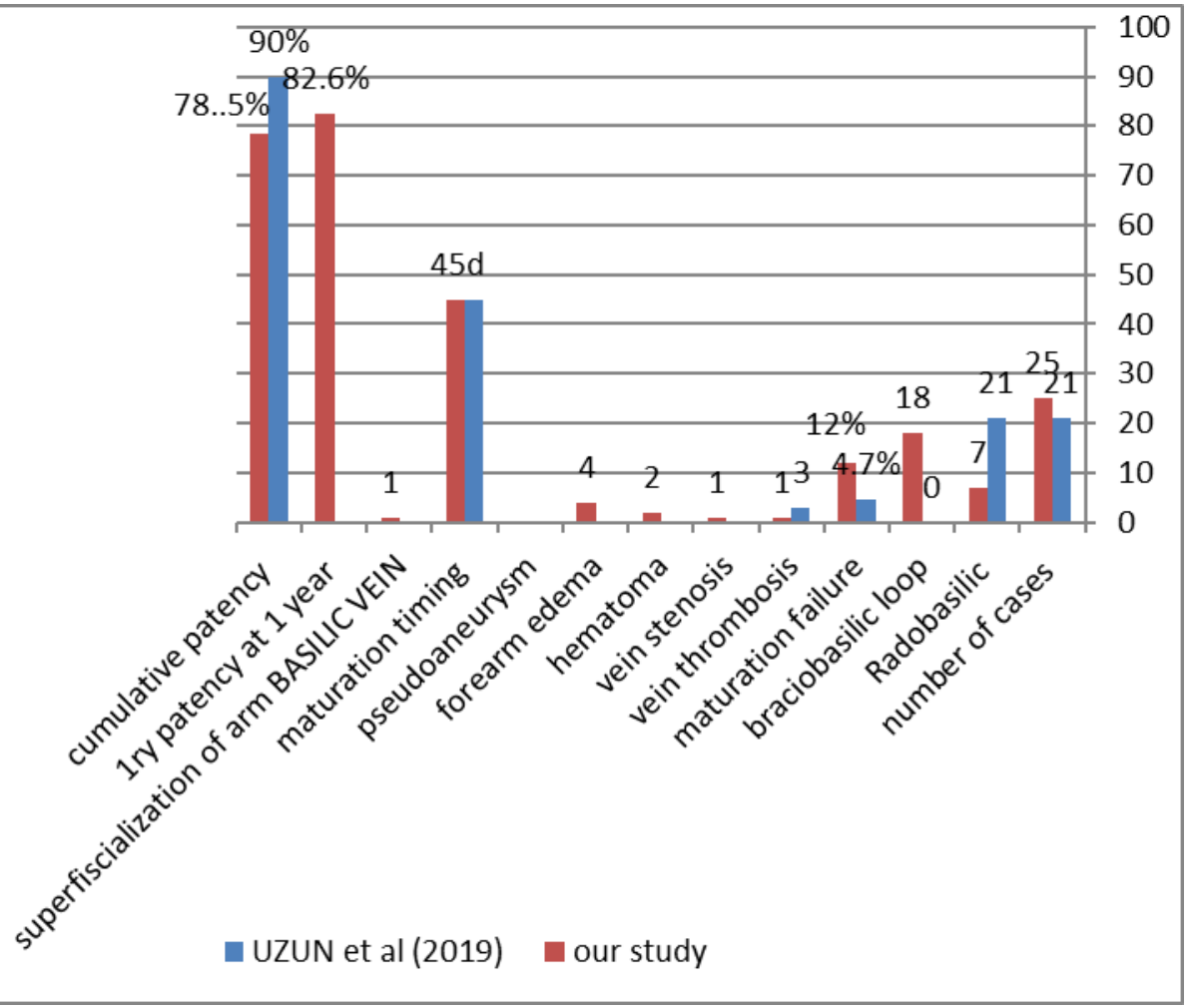

Figure(11): comparison between uzun et al.,(RB AVF ) and our study 


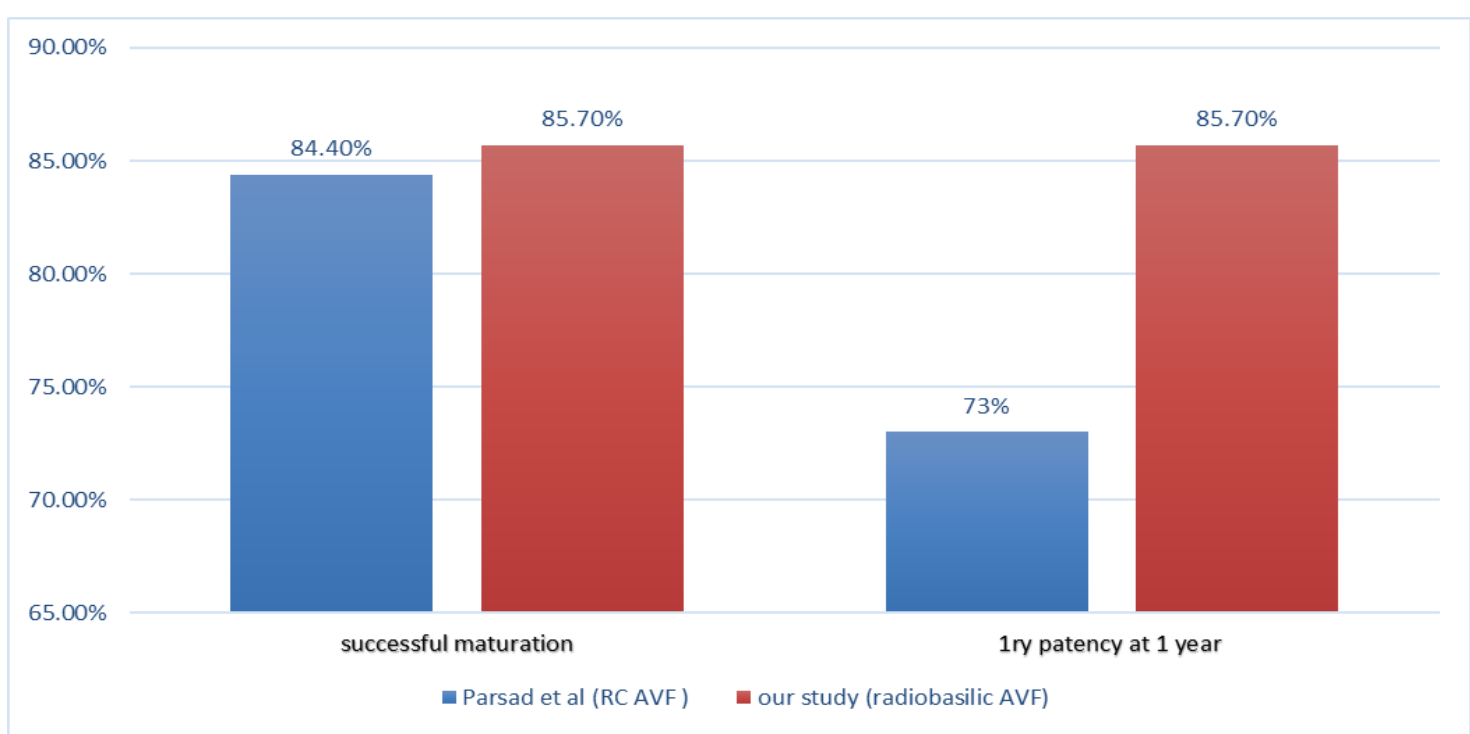

Figure (12) : RC AVF (parsad et al.,) and our RB AVF.

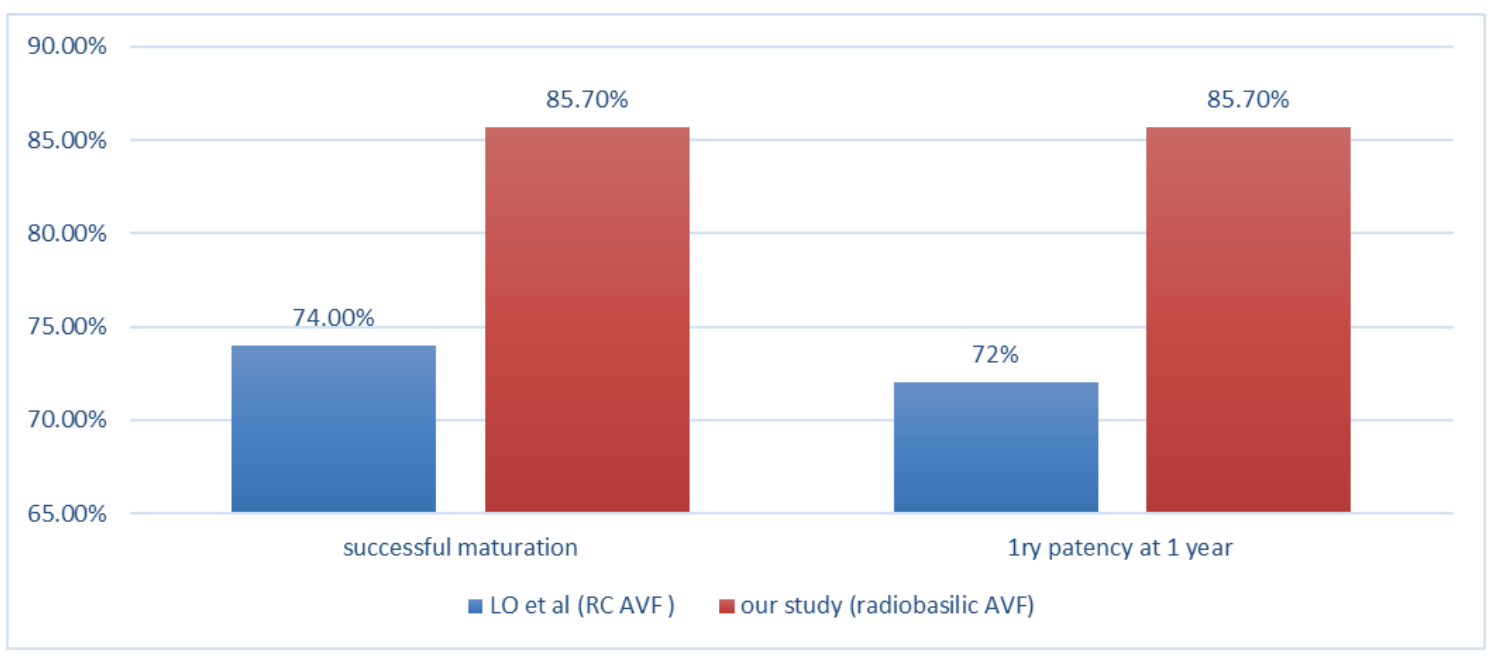

Figure (13) : RC AVF (lo et al.,) and our RB AVF

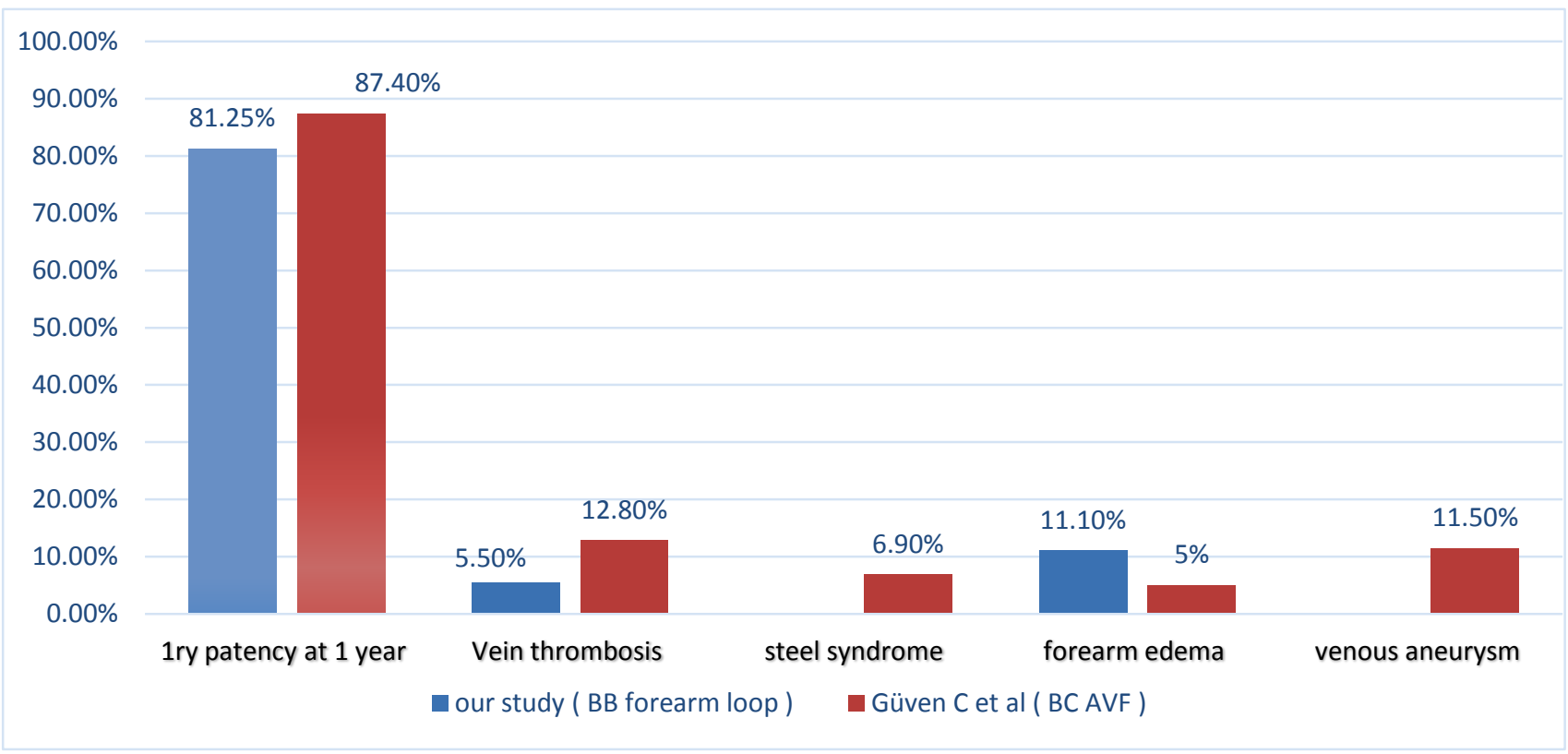

Figure (14) : our study (BB loop) and Guven c et al (BC AVF ). 


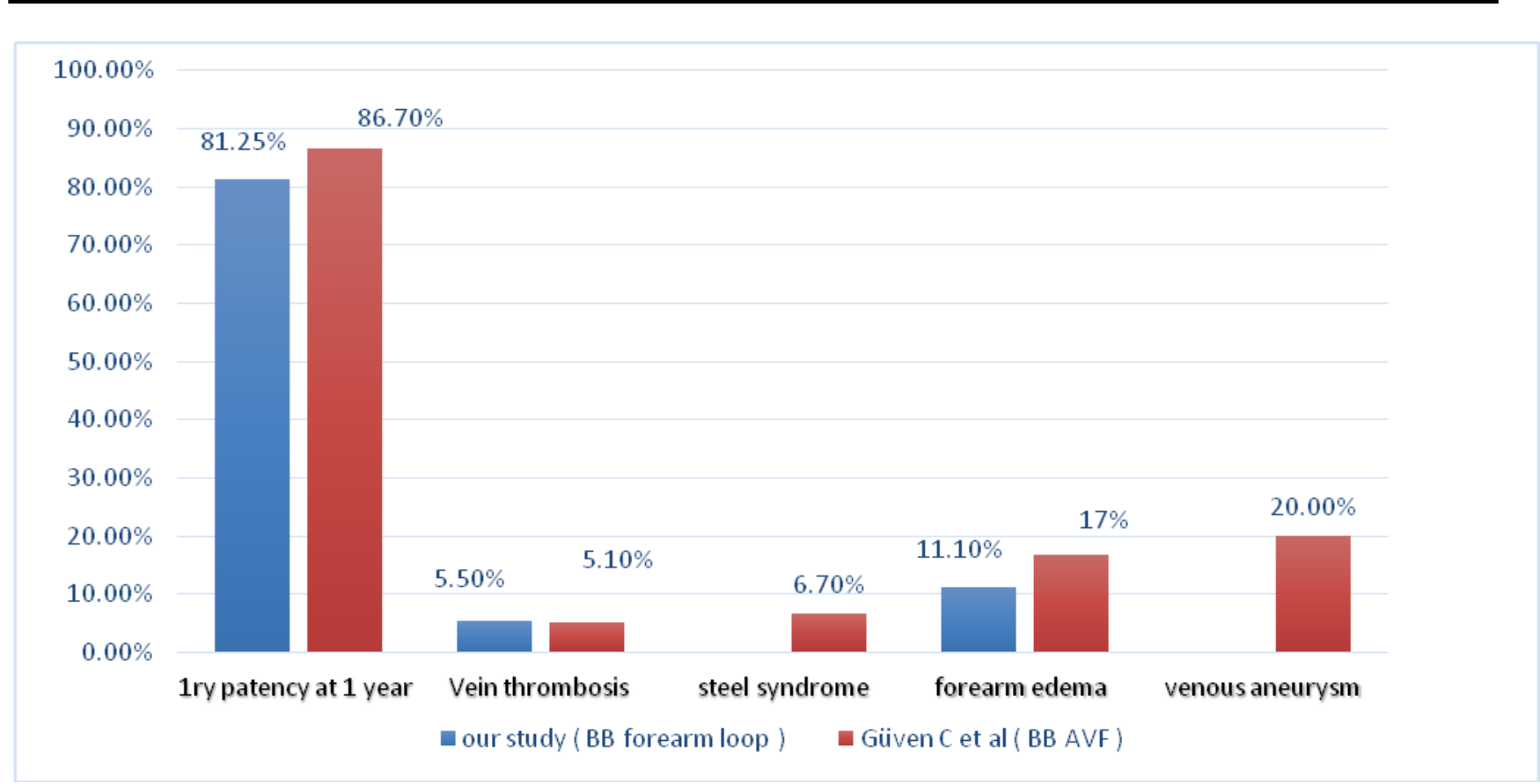

Figure (15) : our study (BB loop) and Guven c et al (BB AVF ).

\section{DISCUSSION :}

it is recommended as Class I indication that patients with a glomerular filtration rate less than $30 \mathrm{~mL} / \mathrm{min} / 1.73 \mathrm{~m} 2$ (Stage 4 chronic kidney disease) should be referred to vascular surgeon for creating haemodialysis access. Therefore, creation of adialysis access should be performed three to six months before initial time of hemodialysis. ${ }^{8}$

It has been proved that autogenous conduits for hemodialysis are more advantageous compared to prosthetic access as they have improved patency rates and lower risk of infection. ${ }^{9}$

Radiocephalic (RC) fistulae remain the first choice access for hemodialysis. The antecubital fossa is recommended as the next site. However, in some patients, forearm basilic vein can be used to create an AVF . As it is rarely used for venipunctures or intravenous lines due to its posteromedial location. Scaring and fibrosis are only found occasionally. ${ }^{10}$ Necessary evaluations which must be performed before placement of a permanent hemodialysis access include physical examination and Duplex USG of the upper extremity arteries and veins.
Arm basilic vein transposition either by one or two stages was discussed and considered as a standard operation for autogenous access, which is often available and kept away by its deep medial position from vein punctures.

$\mathrm{BV}$ transposition in the forearm can be a valuable option, specially for secondary AVFs. It can be a better option before brachiocephalic AVF in extremely obese patients if its diameter greater than $2.5 \mathrm{~mm}$ and has an adequate length allowing us to use it as an AVF tract. ${ }^{11}$

Compared to similar studies, Zeilinski et al, they performed 24 cases with BB loop AVF. maturation failure occurred in $4.2 \%$ of cases . 4 cases had vein thrombosis. 3 had hematomas . 2 cases developed forearm edema and 2 patients had venous pseudoaneurysm. Maturation timing was approximately 40 days .4 cases needed superfiscialization of arm basilic vein . 1ry patency rate at 1 year was $77 \%$ while 2ry patency rate was $81 \%$. figure 10 .

Compared to Uzun HA et al , they performed 21 cases with radiobasilic AVF . maturation faire was 
reported in $4.7 \%$. 3 cases had vein thrombosis . maturation timimg was approximately 45 days . overall patency rates was $90 \%$. figure 11 .

Literature show similar patency rates and complications between forearm basilic vein transposition and distal RC AVF. As for example : Parsad et al ., reported successful maturation of RC AVF as $84.4 \%$. While 1ry patency rate was 73 $\%$ at 1 year.$^{12} \mathrm{LO}$ et al., had Retrospective review of 436 RCAVFs created between 2009 and 2013. 1ry patency rate of studied cases were $72 \% .^{13}$ In our study $85.7 \%$ of patients with RB AVF achieved successful maturation and patency at 1 year is $85.7 \%$. figure 12,13 .

In Literature Arm AVF (BC, BB AVF ) have similar patency rates but less operative time and more complications compared to BB loop AVF .

Guven $\mathrm{C}$ et al., reported 1ry patency of $\mathrm{BC} \mathrm{AVF}$ and $\mathrm{BB}$ AVF at 1 year as $87.4 \% 86.7 \%$ consecutively.$^{14}$ in our study 1ry patency rate of BB looped AVF is $81.25 \%$. However incidence of steel syndrome, venous aneurysms and venous thrombosis were higher in patients with $\mathrm{BC}$ and BB AVFS than BB looped AVF . figure 14,15.

A lot of procedures have been developed to create functional and long-standing vascular access. The FBVT approach has remarkable features: It has similar results to distal AVFs so we can preserve proximal veins for future use we can avoid more complex choices.

FBVT has similar complication rates compared to radiocephalic AVFs as regarding maturation failure, thrombosis, infection, rupture AVF or steel syndrome.

FBVT approach provide suitable place for cannulation, as well as offering more comfort with less steal syndrome than arm brachiobasilic AVFs.
Dissection of forearm basilic vein requires multiple incisions in the forearm compared to distal or proximal AVF which usually are performed using single incision . However wound healing was achieved appropriately with no major complications . forearm edema ,harvesting wound seromas and hematomas can occur following dissection and they can be managed conservatively - Supraclavicular, local and even general anaesthesia may be needed in FBVT . FBV dissection often requires more time than other AVFs .

\section{Conclusion :}

The forearm basilic vein transposition represents avaluable option for autogonous AVF. the transposition of FBV and anastomosis to the radial or brachial artery represents an additional option for selected patients. According to our study FBVT is a tech-nically feasible and very effective surgical procedure for the creation of durable access for haemodialysis, particularly in patients with cephalic vein inadequacy .

We recommend utilization of forearm basilic vein either radiobasilic or looped $\mathrm{BB}$ as avaluable vascular access in case of inadequate cephalic vein preserving proximal veins for future use, delaying usage of prosthetic grafts and avoiding more complications.

\section{Statistical analysis and data interpretation:}

Data were fed to the computer and analyzed using IBM SPSS Corp. Released 2013. IBM SPSS Statistics for Windows, Version 22.0. Armonk, NY: IBM Corp. Qualitative data were described 
using number and percent. Quantitative data were described using mean, standard deviation for parametric data after testing normality using Kolmogrov-Smirnov test. Significance of the obtained results was judged at the (0.05) level.

\section{Data analysis}

Qualitative data :

- Fischer Exact test was used as correction for Chi-Square test when more than $25 \%$ of cells have count less than 5 in $2 * 2$ tables

\section{Quantitative data between groups:}

\section{Parametric tests:}

- Student t-test was used to compare 2 independent groups

\section{References :}

1.Son H., KeeMin S., Min S. Park Y.Ha J., Kim S., 2010: Evaluation of the efficacy of the forearm basilic vein transposition arteriovenous fistula, journal of vascular surgery ,51(3).

2. Srivastava A, Sharma S., 2011: Hemodialysis vascular access options after failed BresciaCimino arteriovenous fistula, indian $\mathrm{j}$ urol, 27(2).

3. Chipde A., Agrawal S., Kalathia J., Mishra U., Agrawal R., 2017: Basilic vein transposition: A viable alternative for multiple failed arteriovenous fistulas - A single center experience, Saudi J Kidney Dis Transpl 28(2).

4.Gameel A., Samir A., Sorour W., 2012: The use of basilic vein transposition in the forearm as an alternative autogenous hemodialysis access,ain shams j surg 5(1)
5. Vadivelu P., kumar JV., Sritharan N., Ilayakumar P., Velladurachi B.,krishna M., devarajan $\quad$ I.,and kumar SP.,2018:EFFICACY OF BASILIC VEIN TRANSPOSITION IN THE FOREARM FOR SECONDARY ARTERIOVENOUS FISTULA CREATION, Int. J. Adv. Res. 6(2).

6. Marsh A. M., Genova R., \& Buicko J. L. (2020). Dialysis Fistula. In StatPearls. StatPearls Publishing.

7. Zielinski M., Inston N., Z Krasinski Z, Gabriel M., and Oszkinis G., 2018: The forearm basilic vein looped transposition fistula as a tertiary option for upper limb vascular access, The Journal of Vascular Access, 19(6).

8. (Schmidli J, Widmer MK, Basile C, de Donato G, Gallieni M, Gibbons CP, et al. Editor's Choice - Vascular Access: 2018 Clinical Practice Guidelines of the European Society for Vascular Surgery (ESVS). Eur J Vasc Endovasc Surg 2018;55:757-818).

9. Murad MH, Elamin MB, Sidawy AN, Malaga G, Rizvi AZ, Flynn DN, et al. Autogenous versus prosthetic vascular access for hemodialysis: a systematic review and metaanalysis. J Vasc Surg 2008;48:34-47).

10. Glowinski J., Malyszko J., \& Gacko M., (2014). Basilic vein transposition in the forearm for secondary arteriovenous fistula. Angiology, 65(4), 330-332.

11. Uzun HA, Çiçek ÖF, Seren M. Transposition of basilic vein in forearm for arteriovenous fistula creation: Our mid-term results. Turk Gogus Kalp Dama 2019;27(4):508-511. 
12. Prasad, N., Thammishetti, V., Bhadauria, D. S., Kaul, A., Sharma, R. K., Srivastava, A., \& Gupta, A. (2019). Outcomes of radiocephalic fistula created by nephrologists. The journal of vascular access, 20(6), 615-620.

13. Joseph Lo, Z., Tay, W. M., Lee, Q., Chua, J. L., Tan, G. W., Chandrasekar, S., \& Narayanan, S. (2016). Predictors of radiocephalic arteriovenous fistulae patency in an Asian population. The journal of vascular access, 17(5), 411-416.

14. Güven, C., \& Uludağ, Ö. (2020). Proximal Arteriovenous Fistulas in Hemodialysis Patients: Advantages and Disadvantages. Cureus, 12(11), e11657. 